\title{
EL PODER DE LO INÚTIL: LAS TECNOLOGÍAS BLANDAS Y LAS BELLAS ARTES
}

\author{
Natalia Vegas Moreno \\ Investigadora y artista independiente
}

\section{Resumen}

Acercamos el concepto de artesanía como conjunto de conocimientos propios del especialista a diferencia de un arte actual sin utilidad en sí mismo, para distinguir lo tecnológico de lo técnico en arte. La posición de superioridad de lo tecnológico frente a lo técnico se revela en su búsqueda de fines, ya sean éstos materiales, ejemplificados en las tecnologías duras, o inmateriales, asumidos en el concepto de tecnologías blandas. La deriva hacia la tecnologización de los dispositivos, ayuda a acercar el peligro de la defensa del producto inmaterial del arte como alineación con el discurso de la sociedad del capitalismo cognitivo.

\section{Palabras clave: TECNOLOGÍA; ARTE; DISPOSITIVO; INMATERIALIDAD;} CAPITALISMO COGNITIVO

\section{THE POWER OF THE USELESS: SOFT TECHNOLOGIES AND FINE ARTS}

\section{Abstract}

We approach the concept of crafts as body of knowledge that belongs to the expert in contrast to a contemporary art without utility in itself, as a way to distinguish technology from technique in art. The superiority position of the technology from the technique discloses in its search for goals, that can be tangible, exemplified in hard technologies, or intangible, attributed to the concept of soft technologies. The drift toward technologization of devices, helps to approach the danger of defending the immaterial art product as an alignment with the discourse of the society of cognitive capitalism.

Keywords: TECHNOLOGY; ART; DISPOSITIF; IMMATERIALITY; COGNITIVE CAPITALISM

\footnotetext{
Vegas Moreno, Natalia. 2016. "El poder de lo inútil: las tecnologías blandas y las bellas artes". AusArt 4(2): 195-204 D0I: 10.1387/ausart. 17105
}

\section{AUSART}




\section{INTRODUCCIÓN: CONFRONTACIONES BLANDO/ DURO Y POSICIÓN DEL ARTE}

De un tiempo a esta parte se llevan relacionando algunos conceptos un tanto polémicos como las tecnologías blandas con las artes - acaso por la desmaterialización que ha sufrido el arte en los últimos siglos- haciendo resurgir ciertas dualidades que parecían olvidadas en el campo de las bellas artes. La relación que se establece entre las tecnologías blandas, también llamadas tecnologías sociales - por tratar con interacciones humanas y protocolos sociales - y las bellas artes como trabajos ambos cuyo objeto es social, podría deberse a nuestra tendencia hacia un pensamiento dualista que mantiene abiertos debates como el que distingue las ciencias de las humanidades (el debate de las dos culturas). De este modo las tecnologías blandas, en firme oposición a las tecnologías duras, estarían relacionadas con las ciencias blandas, en las cuales se cuestiona la rigurosidad y exactitud de las mismas al no utilizar procedimientos cuantificables, objetivables y capaces de producir predicciones. Esto llevaría a organizar en un principio en el bando de las ciencias duras a las naturales y físicas y en el de las blandas a las sociales y humanas, olvidando que este debate del "yo más" (yo más objetivo que tú, yo más exacto) también podría desplazar a unas ciencias duras como menos exactas que otras de su misma categoría. Esta cuestión es manifestada continuamente por el personaje de ficción Sheldon Cooper en la serie de televisión americana The Big Bang Theory, el cual dedicándose a la física teórica desprecia como menores al resto de ciencias como la biología, la geología o la ingeniería: "Ingeniería...donde los trabajadores semicualificados Ilevan a cabo el trabajo de los que piensan y sueñan" (Lorre, Molaro \& Prady 2008)1.

Juan Luis Moraza (2012) distingue cuatro tipos de "ciencia" en la historia del conocimiento humano como son:

(a) ARS; La «ciencia», indiscernible de «arte», identificó en primer lugar un "saber hacer», una sapientia asociada a un «saber» en el que no existe diferencia entre la experiencia sensible y el conocimiento inteligible. (b) LOGOS; La ciencia entendida como sistema ordenado de proposiciones derivadas de principios. (c) TECHNOS; Las ciencias positivas o experimentales. (d) ANTHROPOLOGOS. Las ciencias humanas, que toman a los sujetos como su objeto. La superposición progresiva de estas cuatro modalidades de «ciencia», - -ajustada cada vez a la "centralidad y superioridad» tecnológica $(b, c)$ - , aplicadas incluso sobre las ciencias humanas (d), 
conducen a la noción de «conocimiento» en las «sociedades del conocimiento».

Esta superioridad de lo tecnológico remite a la acepción de "arte" como técnica especializada utilizada durante los siglos XIX y XX $-\mathrm{y}$ que posteriormente pasa a llamarse industria técnica- que es descrita en el Diccionario Akal de Estética con tres características: el empleo de procedimientos reglados, la necesidad de conocimientos propios y la relación con obras concretas. Esta acepción sobrevive en la traducción anglosajona del concepto de tecnología punta que se traduce como state-of-the-art-technology. El concepto de "art" en esta traducción remite al useful art, esto es, a las herramientas y métodos útiles en la práctica de la artesanía, una artesanía que en la época victoriana se usaba como antónimo de las bellas artes y de las artes performativas. Así, encontramos en la artesanía un antecedente de la tecnología como desarrollo máximo de las artes útiles, en relación con la idea de progreso que defienden las ciencias aplicadas y las ciencias naturales. ¿Es entonces el arte actual (arte de las bellas artes) - a diferencia de esta artesanía ya casi extinta y relegada al pasatiempo, el folclore o las terapias creativas - un arte inútil, un arte sin función?, y de tenerla ¿cuál sería la función del arte contemporáneo? Como diría Nicolas Bourriaud "una obra de arte no tiene ninguna función útil a priori, no porque sea socialmente inútil, sino por estar disponible, flexible, 'con proyección al infinito"” (Bourriaud [1998] 2008).

\section{ARTE TECNIFICADO}

Cuando se relacionan las tecnologías sociales con las bellas artes quedamos bloqueados en el concepto de tecnología, preguntándonos si es posible relacionar -y cómo - la tecnología con la práctica artística. Ciertamente, si asumimos la definición de tecnología como conjunto de conocimientos propios de un oficio científicamente ordenados, nos alejamos del saber propio del arte, un saber de saberes que escapa siempre de cualquier posible ordenación y catalogación -y en lo que creemos radica uno de sus mayores potenciales. Continuando con las definiciones de tecnología, aparecen nuevamente los conceptos de utilidad, cuando se describe como un conjunto de herramientas útiles para un ahorro de tiempo y trabajo, así como el de finalidad, al definirse como métodos y/o procesos dirigidos a cumplir unos objetivos. De modo que 
volvemos a ver al arte alejado de esta descripción como útil o con una finalidad de cumplir objetivos.

Es en este punto donde vemos conveniente distinguir entre técnica y tecnología en arte, posicionándonos en que el arte es técnica, supone el desarrollo de una técnica propia y su constante reformulación, pero no tecnología; ya que esa misma necesidad técnica de la constante puesta en cuestión de su técnica, hace improductivo una conjunción de su conocimiento.

Regresando a las definiciones, descubrimos en la distinción entre técnica y tecnología algo que remite de nuevo a esa actualidad de la superioridad tecnológica que mencionaba Moraza, así como a relacionar con ello el distanciamiento de unas artes aplicadas y unas artes estéticas. La técnica se define como el conjunto de procedimientos de que se sirve una ciencia, arte o habilidad, como acción y método de ejecución. De modo que la historia del progreso humano, es también una historia de la técnica. Sin embargo existe un punto en la historia en la que esta técnica evolucionó hacia una tecnología, donde el conjunto de procedimientos pasa a ser conjunto de los conocimientos propios de un oficio, añadiéndose un grado superior a la enseñanza técnica, la de los ingenieros y especialistas. Éstos se basan en el entendimiento, en llegar a saber por medio de la inteligencia y el ingenio la naturaleza de una cosa, lo que remite a la epistemología como estudio del conocimiento científico, ligada a ese grado "superior" de enseñanza técnica. Así, en esta división entre aquellos que operaban con la técnica de manera directa y los que aplican los conocimientos derivados de estas operaciones para un supuesto perfeccionamiento de la misma, se observa una evolución hacia una tecnologización coincidente con la superioridad del "logos" y el "technos" mencionados por Moraza. En lo que atañe al arte, esta tecnologización llevaría a una deriva desde la creación, la acción y la ejecución, al puro conocimiento, a la inmaterialidad y al ablandamiento (en referencia a las tecnologías blandas cuyo objeto es inmaterial), propio de un trabajo en la línea del capitalismo cognitivo.

\section{DISPOSITIVOS DE PODER, DEL TECNICISMO A LA TECNOCRACIA}

La modernidad es el florecimiento de la confianza y las utopías del cambio, pero también de las articulaciones de nuevos procedimientos de control. Si 
bien comentábamos el cambio de la enseñanza técnica hacia su especialización, podríamos situarla en Europa con la apertura de la primera escuela de ingeniería en 1747, la École des Ponts et Chaussés en París. Este dato no resulta baladí en su coincidencia con el nacimiento de los primeros museos de arte, lugares especializados en arte que como tales son de creación moderna ${ }^{2}$, como el Hermitage de San Petersburgo de 1764 o el Louvre de París de 1793. Estos lugares denotan la necesidad de una especialización y división de los saberes en pro de una búsqueda de la libertad y la emancipación universales, defensas propias del proyecto moderno ${ }^{3}$. Pero más interesante que esta relación es que ambos sucesos podrían estar enmarcados en el nacimiento de lo que Michel Foucault Ilama el poder disciplinario. Situándolo en el siglo XVIII, este autor lo distingue del clásico poder soberano, en que el disciplinario utiliza mecanismos de poder adaptados a la vigilancia y el adiestramiento. Esta combinación de técnicas para el control parece tener su resultado más temible en el desmantelamiento de los dispositivos hacia su inmaterialidad e invisibilidad que, como en el caso de las tecnologías sociales, al ejercer su poder desde esa indeterminación difícilmente detectable, las volvería más poderosas.

Pero al margen de su estado, gaseoso, líquido, sólido, duro, blando, la tecnología ha sido desde sus inicios un dispositivo, ya que esa ordenación del conocimiento ha estado dirigida a cumplir unos objetivos, con lo cual nunca podría estar al margen del poder. Como diría Foucault (citado por Agamben 2011), el dispositivo tiene una función estratégica dominante indisociable del poder, y toma formas variadas desde los discursos, las instituciones, las habilitaciones arquitectónicas, las decisiones reglamentarias, las leyes, las medidas administrativas, los enunciados científicos, las proposiciones filosóficas, morales, filantrópicas. En fin, entre lo dicho y lo no dicho, he aquí los elementos del dispositivo. El dispositivo mismo es la red que tendemos entre estos elementos.

Curiosamente, la finalidad tecnológica de la satisfacción de las necesidades esenciales humanas se evidencia como una utopía más cuando vemos que es el propio poder capitalista el que mantiene en estado infinito esas necesidades. Sin embargo esto no ha frenado el tecnicismo, el cual seguimos fomentando en una sociedad que apuesta por el uso del medio como mensaje y masaje. Este tecnicismo promueve la tecnocracia, un gobierno del para, para obtener resultados, para manipular, para comprobar, con una acción orientada siempre a resultados cuantificables, útiles. El arte en cambio, siguiendo las palabras de Jon Mikel Euba, habría que situarlo en el por y no en el para. 
Que cada vez sea más complicado acertar a distinguir entre el dispositivo como límite de la representación y el objeto artístico, como apuntaría Moraza, tendrá que ver con esa ruptura de las categorías que como describía Rosalind Krauss llevaría hacia el campo expandido de las artes. Si lo que Krauss advertía con la expansión de las categorías lo entendiésemos como la abolición de las categorías incurriríamos en un pensamiento idealista, ya que cuando el artista moderno desbancó el pedestal no eliminó el dispositivo sino que lo desplazó y lo multiplicó.

La estética relacional apunta que el problema del arte ya no estará en desplazar sus límites, sino en "poner a prueba los límites de resistencia del arte dentro del campo social global” (Bourriaud [1998] 2008). El problema de estas reivindicaciones estará en llegar al punto de sustituir al arte por la vida, con el peligro que supone la indistinción entre representación y realidad. El compromiso con la representación en arte es un compromiso técnico ineludible ya que, pese a que entendamos en esa construcción cierta inevitable condición de alienación (por su hecho ficcional y simbólico), será esta misma circunstancia la que permita mantener la necesaria barrera para ser con los demás. De modo que en la época contemporánea todas esas cuestiones que en arte se reivindicaban como valores como Las nuevas tecnologías, la negación de la autoría, las estéticas impersonales, la discursividad, laconceptualización extrema y la contextualidad, parecen liberar al arte de sí mismo, pero a costa de convertirlo en sumiso del conocimiento tecnocéntrico de las sociedades del conocimiento, y las industrias de la cultura visual. Por ello no pueden considerarse como alternativas a las formas tradicionales de arte, sino desplazamientos hacia el poder de las empresas tecnológicas y sacrificio de los compromisos de singularidad propios de la excelencia artística (Moraza 2012).

El proyecto moderno de la formación de un metalenguaje universal traslada los métodos científicos al arte, buscando una lógica epistémica en una praxis ilógica y subjetiva. Así, Fredric Jameson diferenciará obras modernas y posmodernas por su "contenido de verdad del arte, su pretensión de poseer alguna verdad o valor epistemológico" (Jameson citado por Owens 2002), pretensión ésta que no pasará de una ilusión moderna, así como de una fe en el conocimiento científico. Pero ante "la aplicabilidad y la objetividad de la ciencia, el arte no ofrece una simulación de conocimiento, sino una experiencia metafórica y real de las complejidades ininteligibles" (Moraza 2012). El arte se mantiene en una búsqueda constante y puesta en cuestión de su propia técnica de búsqueda, es por ello que el arte más bien sería lo que no se puede tecnologizar, lo que escapa a la tecnología, ya que no se podrían aplicar pro- 
tocolos para una reordenación de lo "insabido". Porque como explica Txomin Badiola, aunque el artista asume completamente estar sujeto a fuerzas que le exceden, está al mismo tiempo implícita su obligación de tomar las riendas y de definir una técnica que precisamente le permita circular «por donde no se sabe y hacia donde no se sabe». Por técnica me refiero al elemento praxiológico, a los protocolos del proceder y no a lo que la técnica tendría de expresión inmediata en la acción de una voluntad y desde luego tampoco a lo que serían las técnicas específicas de las artes (Badiola 2006).

Y es esta técnica la que interesa al artista, la que pone a interrogar al propio deseo y que como puntualiza Badiola no puede encontrarse en los libros.

\section{EL PODER DE LO INMATERIAL}

Como ya anunciaba Jose Luis Brea, el sector servicios ha ido dando paso al cuarto sector, el del trabajo inmaterial, un trabajo que tiene como finalidad esa que resonaba a utopía cuando hablábamos de las tecnologías; la satisfacción de necesidades, aunque esta vez se englobarían en el orden de lo psíquico, lo espiritual, el sentido o el deseo. Este producto intangible, que se describe como propio de las tecnologías sociales, tiene el peligroso valor de que no conlleva pérdida para el emisor ya que la transformación de un conocimiento, a diferencia de una mercancía, no hace que éste desaparezca del que lo transmite.

Definitivamente: hablamos de sociedades del conocimiento, o incluso del capitalismo cultural, para designar una fase avanzada de desarrollo del capitalismo en la que el saber, el conocimiento, o incluso la propia esfera de lo cultural [...] se sitúan en el centro mismo de los procesos productivos, generadores de riqueza.

(Brea 2007)

En este capitalismo cultural el arte se convierte en una de las industrias especulativas más deseables, ya que como asegura Jeremy Rifkin "el $20 \%$ de la población estadounidense gasta ya más del $50 \%$ de sus ingresos en acceder a experiencias culturales", y como continúa Brea "La previsión de la mayoría de los autores coincide en señalar que la importancia adquirida por este nuevo sector industrial continuará creciendo en las próximas décadas, hasta conver- 
tirse en el motor principal de las nuevas sociedades" (2007). Este consumo de productos inmateriales ha conseguido desplazar el valor material patrimonial del arte hacia una inmaterialidad peligrosamente alineada con el capitalismo cognitivo. "Al artista ahora se le considera como un investigador que desarrolla procesos dentro de los cuales no sólo produce objetos, sino también conocimiento, lo que se entiende por resultados inmateriales que toman generalmente forma discursiva". (Jaio 2010).

De modo que ese capitalismo cognitivo de productos intangibles se evidencia como dispositivo de redistribución de afectos y organización de las representaciones, cómo describiría Brea, en extraña consonancia con unas tecnologías blandas, unas tecnologías del poder que ahora se esconden bajo una piel de cordero para invisibilizar su control sobre nosotros. Que no veamos al lobo, sin embargo, no quiere decir que no esté.

Esa megaindustria [...] recubre el mundo de una omnipresencia cuya función no es otra que esa desplazada de lo cultural -que consiste en producir al individuo, en construirle como personaje, en proporcionarle argumentos y narrativas de individuación, de reconocimiento de pérdida y distinción en contextos de comunidad, de socialización. [...] lo cultural deja de ser puente de relación con el pasado o las tradiciones, [...] para convertirse sobre todo en dispositivo y argumento de producción de individuación, en macrofábrica de las ficciones que entrelazan el imaginario de una vida propia, en gigantizada industria de la subjetividad.

(Brea 2007)

\section{CONCLUSIÓN: ¿QUÉ PODER LE QUEDA AL ARTE?}

Tras la caída de los relatos que sostenían la mirada — dios, patria, razón, etcel arte pasó de una lucha contra lo Simbólico, contra el esquema establecido, a una autorreferencialidad. Sin embargo esto no quiere decir que haya abandonado su combate contra los dispositivos de poder, ni mucho menos que lo haya ganado. En un momento actual en el que el capitalismo cognitivo quiere imponer unos protocolos en búsqueda y control del conocimiento, el arte se 
presenta como el recurso ideal hacia la desprotocolarización y la desorganización.

El deseo de sentido, propio del humano, puede haberse convertido en uno de los mayores motores para alimentar ese capitalismo cognitivo, una búsqueda infinita que mantiene a la humanidad trabajando. Es la misma búsqueda que pese a saberse fracasada, como diría Badiola, pone a trabajar al artista. Pero la obra de arte genera un plus, un más allá de lo que el artista busca, que desorganiza y reconvoca sus espectativas continuamente. "El sentido es algo tan fatal para el hombre que, en cuanto libertad, el arte parece ocuparse, sobre todo en el presente, no de fabricar sentido sino por el contrario de suspenderlo; de construir sentidos pero no de llenarlos exactamente" (Barthes [1963] 1983). Es por ello que la obra de arte no tendría sentido en sí misma, no tendría función útil en sí, siendo esto mismo su potencial, el potencial de convocar cualquier sentido y cualquier utilidad.

\section{Referencias}

Agamben, Giorgio. 2011. “¿Qué es un dispositivo?”. Traducción de Roberto J. Fuentes Rionda. Revista Sociológica 73(26): 249-64. http://www.revistasociologica.com.mx/pdf/7310.pdf

Badiola, Txomin. 2006. "Hay veces en las que uno tiene que poner en escena su propio fracaso". En Rêve sans fin (la técnica): 10 de octubre-11 noviembre, coordinación Juan R. Rodríguez. Madrid: Galería Soledad Lorenzo

Barthes, Roland. (1963) 1983. "Sobre el cine", declaraciones recogidas por Michel Delahaye \& Jacques Rivette. En El grano de la voz: Entrevistas 1962-1980, traducción de Nora Pasternac. México: Siglo XXI. Originalmente en Cahiers du Cinéma 147, sept. 1963

Bourriaud, Nicolas (1998). 2008. Estética relacional. Traducción de Cecilia Beceyro \& Sergio Delgado. Buenos Aires: Adriana Hidalgo

Brea, Jose Luis. 2007. "E-ck [capitalismo_cultural_electrónico]". En Cultura_RAM: Mutaciones de la cultura en la era de su distribución electrónica. Barcelona: Gedisa

Jaio Atela, Miren. 2010. "Nuevas versiones del par 'estética e ideología': El contexto vasco como caso de estudio del paradigma cultural iniciado a finales del siglo XX". Trabajo Suficiencia Investigadora, Univ. País Vasco, Dpto. Historia del Arte y de la Música

Lorre, Chuck, Steven Molaro \& Bill Prady. 2008. "The Jerusalem Duality". The Big Bang Theory temporada 1, episodio 12, emitido el 14 abril. Burbank, CA: Warner Bros

Moraza, Juan Luis. 2012. "Arte en la era del capitalismo cognitivo". Ponencia de las jornadas EI arte en la Sociedad del Conocimiento, Eusko Ikaskuntza \& Facultad de Bellas Artes UPV/ EHU. Madrid: Reina Sofía. http://www.museoreinasofia.es/sites/default/files/banner/descargas/arte_en_la_era_del_capitalismo_juanluismoraza.pdf

Olmo Alonso, Saioa \& ColaBoraBora (grupo). 2016. “\#Tecno_blandas”. Acceso 29 agosto. http://www.tecnologiasblandas.cc/ 
Owens, Craig. 2002. "El discurso de los otros: Las feministas y el posmodernismo". En La posmodernidad, Jean Baudrillard et al.; selección y prólogo de Hal Foster; traducción, Jordi Fibla. Barcelona: Kairós

Souriau, Étienne. (1990) 1998. Diccionario Akal de Estética. Traducción de Ismael Grasa Adé. Madrid: Akal

Vegas Moreno, Natalia. 2015. "Aproximaciones a una experiencia de malestar contemporáneo. Entre la ausencia de símbolo-arte y el encuentro con las malas formas en la obra de Jon Mikel Euba". Tesis Univ. País Vasco, Dpto. Escultura

\section{Notas}

1 "Ingeniería segun Sheldon Cooper". Vídeo de Youtube, 1:36, Subido el 17 abr. 2010 https:// www.youtube.com/watch?v=IgnnYGAuDVw [Extracto de The Jerusalem Duality, temporada 1, episodio 12 de The Big Bang Theory]

${ }^{2}$ Cuando hablamos de modernidad en este punto nos referimos a la datación del cambio histórico que tuvo lugar con la Revolución Industrial hacia el siglo XVIII.

${ }^{3} \mathrm{Si}$ bien el proyecto moderno al que nos referimos es un concepto filosófico que designa un periodo histórico-social fechado entre los siglos XIX y XX, éste tendrá su antecedente en los cambios históricos acontecidos previamente y que dan entrada a la Edad Contemporánea. 